\title{
CyberKnife stereotactic radiosurgery for the treatment of symptomatic vertebral hemangiomas: a single-institution experience
}

\author{
${ }^{*}$ Michael Zhang, MD, Yi-Ren Chen, MD, MPH, Steven D. Chang, MD, and Anand Veeravagu, MD \\ Department of Neurosurgery, Stanford University, Palo Alto, California
}

\begin{abstract}
OBJECTIVE Symptomatic vertebral hemangiomas (SVHs) are a very rare pathology that can present with persistent pain or neurological deficits that warrant surgical intervention. Given the relative rarity and difficulty in assessment, the authors sought to present a dedicated series of SVHs treated using stereotactic radiosurgery (SRS) to provide insight into clinical decision making.
\end{abstract}

METHODS A retrospective review of a single institution's experience with hypofractionated radiosurgery for SVH from 2004 to 2011 was conducted to determine the clinical and radiographic outcomes following SRS treatment. The authors report and analyze the treatment course of 5 patients with 7 lesions, 2 of which were treated primarily by SRS.

RESULTS Of the 5 patients studied, 4 presented with a chief complaint of pain refractory to conservative measures. Three patients reported dysesthesias, and 2 reported upper-extremity weakness. Following radiosurgery, 4 of 5 patients exhibited improvement in their primary symptoms ( 3 for pain and 1 for weakness), achieving a clinical response after a mean period of 1 year. In 2 cases there was 20\%-40\% reduction in lesion size in the most responsive dimension as noted on images. All treatments were well tolerated.

CONCLUSIONS SRS for SVH is a safe and feasible treatment strategy, comparable to prior radiotherapy studies, and in select cases may successfully confer delayed decompressive effects. Additional investigation will determine future patient selection and how conformal SRS treatment can best be administered.

https://thejns.org/doi/abs/10.3171/2016.9.FOCUS16372

KEY WORDS symptomatic vertebral hemangioma; spine; CyberKnife; hypofractionation; stereotactic radiosurgery

$\mathrm{V}$ ERTEBRAL hemangiomas are benign lesions of the spinal column that are often incidentally found. Most are managed by observation due of their asymptomatic course, although $1 \%$ may eventually result in clinical symptoms. ${ }^{11}$ The usual presenting symptom is one of axial back or thoracic pain, likely a consequence of an extramedullary component inducing neuroforaminal or canal stenosis. ${ }^{5,14}$ Less frequently, these lesions are known to cause worrisome cord compression with subsequent weakness or bowel and bladder symptoms. ${ }^{2,13,24,26,27}$ Patients with symptomatic vertebral hemangiomas (SVHs) may benefit from intervention..$^{11}$ The options have widely included decompression, embolization, vertebroplasty, radiation therapy, and, less frequently discussed, radiosurgery. ${ }^{8,11,12,18}$
There are currently several studies in the literature discussing the response of SVH to radiation therapy. Heyd et al. reported in one of the most successful series of its size: a 40-year, multicenter review of 84 patients who were treated with a median dose of 34 Gy to achieve a 90.5\% symptom response rate. ${ }^{14}$ More recently, Miszczyk and Tukiendorf reported a single-center experience with 101 patients, where there was a more conservative $78.4 \%$ response rate..$^{18}$ Smaller cohorts have reported similar success with radiotherapy rates ranging from $82 \%$ to 93\%. $3,22,23,27$

Although radiotherapy has been a mainstay in the treatment algorithm for SVH, the less studied stereotactic radiosurgery (SRS) is known to achieve a superior dose drop-off that may confer clinical and safety benefits. ${ }^{10,17,21}$

ABBREVIATIONS BED = biologically effective dose; $\mathrm{nBED}$ = normalized BED; SRS = stereotactic radiosurgery; SVH = symptomatic vertebral hemangioma. SUBMITTED September 1, 2016. ACCEPTED September 27, 2016.

INCLUDE WHEN CITING DOI: 10.3171/2016.9.FOCUS16372.

* Drs. Zhang and Chen contributed equally to this work. 
CyberKnife radiosurgery is one such SRS modality that can successfully administer high-dose radiation to within 1-mm accuracy for mobile spine targets. ${ }^{1,6,7,21}$ Its incorporation of a frameless technique, real-time imaging, and adaptive beam pointing during treatment has offered an alternative to more invasive immobilization techniques while maintaining high-accuracy targeting in the spine.

Despite these technical advances, very little has been described regarding the success of SRS for vertebral hemangioma. Only one study by Sohn et al. has detailed the use of radiosurgery for the treatment of a spinal hemangioma presenting with worsening radiculomyelopathy following a decompression and partial resection of the lesion that was limited by intraoperative bleeding. ${ }^{25}$ Additional reporting on SVHs that have been treated using radiosurgery has largely been amalgamated with other or benign pathologies.12,18,25 Thus, we present a detailed account of the first series of SVH treated by hypofractionated radiosurgery.

\section{Methods \\ Patients}

Patients treated for SVH at Stanford Health Care between 2004 and 2011 were identified, and patient characteristics including age, sex, comorbidities, site of lesion, clinical presentation, clinical outcome, radiographic outcome, length of follow-up, and treatment planning values were collected. Treated lesions included 5 thoracic, 1 cervical, and 1 lumbar lesion. Findings are listed in Table 1. Clinical and radiographic follow-up were requested at 3 and 6 months and every year after the date of surgery.

\section{Radiosurgery Treatment Planning}

The tumor volume ranged from 0.6 to $70.9 \mathrm{~cm}^{3}$, the prescription dose from 15 to $27.5 \mathrm{~Gy}$, the prescription isodose from $72 \%$ to $83 \%$, and the maximum dose from 18.1 to $36.7 \mathrm{~Gy}$. Lesions were treated in fractions ranging from 1 to 5 sessions (median 1 session). The biologically effective dose (BED) was calculated using the formula $n d(1+$ $d / \alpha / \beta)$. The 2-Gy biologically equivalent dose, normalized BED (nBED) was calculated by dividing the BED by (1 $+d / \alpha / \beta)$ where $d$ is 2 Gy. The $\alpha / \beta$ for hemangioma was conservatively estimated to be $4.0 \mathrm{~Gy}$. Treatment variables are provided in Table 2.

\section{Radiosurgery Preparation}

Prior to treatment, patients with cervical lesions underwent fabrication of an Aquaplast facemask for immobilization (WFR/Aquaplast Corp.). For thoracic as well as lumbar lesions, the patients rested in an Alpha Cradle (Smithers Medical Products). Some cases involving thoracic and lumbar vertebral lesions also required implantation of percutaneous fiducial markers in the posterior bony elements to provide additional radiographic landmarks and aid with movement compensation as previously described..$^{21}$

Patient imaging was performed using high-resolution thin-slice CT with a GE Light Speed 8i or 16i scanner (General Electric Medical Systems) after administration of Omnipaque intravenous contrast (iohexol, $350 \mathrm{mg}$ io- dine/ml; Nycomed, Inc., GE Healthcare). Subsequent stereotactic MRI studies were obtained and fused to the stereotactic CT scan to improve target identification.

The team of physicians, comprising a neurosurgeon, a radiation oncologist, and a radiation physicist, performed tumor delineation, dose selection, and planning. Informed consent for treatment was obtained from all patients, and IRB approval was granted. The tumor volume was outlined, and inverse treatment planning was performed to achieve a highly conformal treatment plan with efforts to minimize dosing to the spinal cord.

\section{Results \\ Case Reports}

Case 1

A 72-year-old man presented with right-hand weakness and arm pain 1 week after shoulder surgery in May 2011 to repair what was thought to be a torn rotator cuff derived from a fall 6 months earlier. He had been diagnosed with chronic regional pain syndrome and had been managed with opiates. CT and MRI from additional workup identified cord compression at the T1-2 level due to a vertebral hemangioma (Fig. 1). The patient underwent decompression and fusion in October 2011, followed by adjuvant SRS for the residual lesion for ongoing pain. Durable pain improvement was noted by 1 year, and by 2 years, the patient no longer required opiates.

\section{Case 2}

A 30-year-old woman had acutely severe, worsening midthoracic radicular pain in January 2004. There was also T8-9 dermatomal patchy numbness. The patient had history of a snowboarding accident with injury to her left shoulder 1 year earlier, breast augmentation, a motor vehicle accident, and migraines. Initial MRI identified a midline sagittal T-9 lesion with no appreciable mass effect; thus, it was first suggested that she proceed with conservative observation. The lesion was considered too posterior for safe vertebroplasty. At 13 months of follow-up her pain had worsened, and she opted for SRS. At 6 months, given a lack of response, SRS was repeated. At the next 6-month follow-up, there was a reported $30 \%$ reduction in pain as the patient continued to work with the pain management team. Subsequent care included frequent minor procedures as well as vertebroplasty at an outside hospital without additional benefit.

\section{Case 3}

A 46-year-old woman had initially presented to an outside facility for embolization of a large S1-3 hemangioma in 1995. There was persistent back pain and decreased right foot sensation in 2002. Workup at this time identified an additional L-2 lesion involving the sacrum, as well as additional clavicular and thoracic lesions. The patient underwent initial embolization. Recurrent back pain brought her to our institution in 2004, where CT imaging confirmed a persistent L-2 lesion involving the sacrum. She underwent repeat embolization with adjuvant SRS. A second round of SRS was performed for her thoracic hemangiomas. She underwent radiological follow-up and 


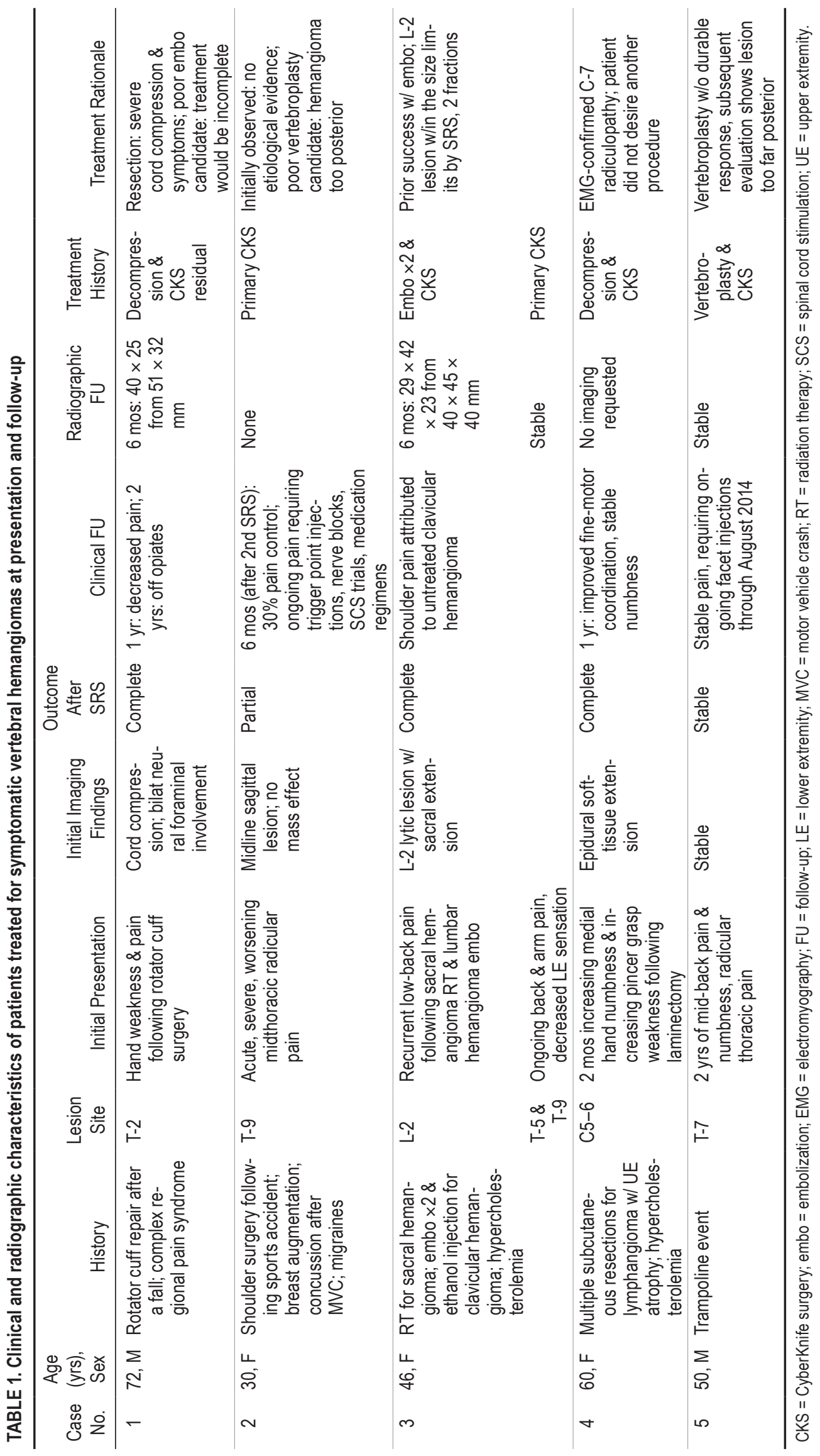


TABLE 2. CyberKnife radiosurgical treatment parameters for 5 patients with 7 SVHs

\begin{tabular}{cccccccccc}
\hline $\begin{array}{c}\text { Case } \\
\text { No. }\end{array}$ & Site & $\begin{array}{c}\text { FU } \\
(\mathrm{mos})\end{array}$ & $\begin{array}{c}\text { No. of } \\
\text { Fractions }\end{array}$ & $\begin{array}{c}\text { Prescription } \\
\text { Dose }(\mathrm{Gy})\end{array}$ & $\begin{array}{c}\text { Max Dose } \\
(\mathrm{Gy})\end{array}$ & $\begin{array}{c}\text { Tumor Vol } \\
\left(\mathrm{cm}^{3}\right)\end{array}$ & $\begin{array}{c}\text { Prescription } \\
\text { Isodose } \%\end{array}$ & $\begin{array}{c}\text { Conformity } \\
\text { Index }\end{array}$ & $\begin{array}{c}\text { Modified } \\
\text { Conformity Index }\end{array}$ \\
\hline 1 & T1-2 & 44 & 5 & 27.5 & 36.67 & 70.90 & 75 & 1.31 & 1.18 \\
\hline 2 & T-9 & 27 & 1 & 15 & 18.07 & 0.60 & 83 & 1.64 & 1.47 \\
\hline 3 & L-2 & 55 & 2 & 18 & 23.38 & 36.41 & 77 & 1.34 & 1.25 \\
\hline & T-5 & & 1 & 18 & 24.32 & 0.84 & 74 & 1.73 & 1.58 \\
\hline & T-9 & & 2 & 18 & 25 & 1.92 & 72 & 1.59 & 1.48 \\
\hline 4 & C5-6 & 25 & 1 & 18 & 22.5 & 2.93 & 80 & 1.47 & 1.42 \\
\hline 5 & T-7 & 75 & 1 & 18 & 22.5 & 2.77 & 80 & 1.91 & 1.73 \\
\hline & Median & 44 & 1 & 18 & 23.38 & 2.77 & 77 & 1.59 & 1.47 \\
\hline & Min & 25 & 1 & 15 & 18.07 & 0.60 & 72 & 1.31 & 1.18 \\
\hline
\end{tabular}

was found to have regression of her hemangiomas with no further interventions required.

\section{Case 4}

A 60-year-old woman with a history of congenital lymphangioma requiring multiple subcutaneous resections and with baseline left upper-extremity atrophy presented with electromyography-confirmed C-7 radiculopathy in May 2007. MRI identified neuroforaminal involvement of an epidural C5-6 hemangioma, and she subsequently underwent decompression and fusion. There was initial improvement before new medial hand numbness and increased pincer grasp weakness was reported in the month following surgery. She subsequently underwent SRS. At 1-year follow-up there was improved fine motor coordination and stable numbness.

\section{Case 5}

A 50-year-old man presented with 2 years of primarily mid-back pain with thoracic radiation. A hemangioma localized to the T-7 vertebral body, without associated central canal or foraminal stenosis, was discovered during workup (stable spine according to the Spinal Instability Neoplastic Score criteria). Prior to our encounter, he had undergone several epidural injections and radiofrequency ablations, none of which were of benefit. The patient underwent SRS, which did not improve the patient's pain. He continued to work with the pain management team, adjusting medications and undergoing minor procedures.

\section{Clinical Results}

Patients treated for SVH presented with a wide range of symptoms, with 4 of 5 patients identifying some degree of pain refractory to conservative measures including significant opiate usage and pain clinic interventions. Three patients had dysesthesias, and 2 exhibited upper-extremity weakness.

Following radiosurgery, 4 of 5 patients exhibited some degree of symptomatic improvement: 3 for pain and 1 for weakness. Among the 4 patients who presented with pain, 2 patients (Cases 2 and 5) pursued ongoing outpatient management with minor procedures, including injections, nerve block, spinal cord stimulation trials, and face injec- tions. The patients in Cases 1 and 4 did not require opiates at last follow-up. The time to clinical response for these individuals occurred over an average period of 1 year. One of these patients (Case 4) did not present with primary concerns of pain, but this patient also experienced improvement in strength within 1 year of follow-up.

\section{Radiographic Results}

Prior to SRS, 3 patients demonstrated cord compression, and 1 additional patient presented with neuroforaminal involvement, all with clinical findings consistent with the dermatomal or myotomal distribution of their SVHs. During follow-up, 4 of 5 patients were radiographically monitored. On postoperative MR images, 2 patients (Cases 1 and 3) exhibited shrinking of their tumor's paraspinal component compared with preradiosurgical scans at 6 months, with $20 \%-40 \%$ reduction in the size within a given dimension. One patient did not receive follow-up imaging due to significant improvement in the motor examination results following treatment.

\section{Treatment Considerations}

All patients were considered for alternative interventions prior to CyberKnife surgery, and 4 of 5 patients underwent a combination of surgical procedures. Two patients (Cases 1 and 4) underwent an initial decompression resection, one with partial resection and the other without. These patients underwent open resection because of their localizable compression and symptoms. Two patients (Cases 3 and 5) considered alternatives prior to SRS, including repeat embolization and vertebroplasty Following primary radiosurgery with partial response, 1 patient (Case 2) ultimately sought vertebroplasty at an outside institution without additional benefit. During follow-up, the patient in Case 4 underwent multiple CyberKnife treatments for her thoracic hemangiomas, given her positive and safe response to treatment to her lumbar lesion. All treatments were well tolerated without subsequent symptoms associated with radiotherapy to the spinal cord.

\section{Discussion}

Radiosurgery is rarely considered a primary treatment 


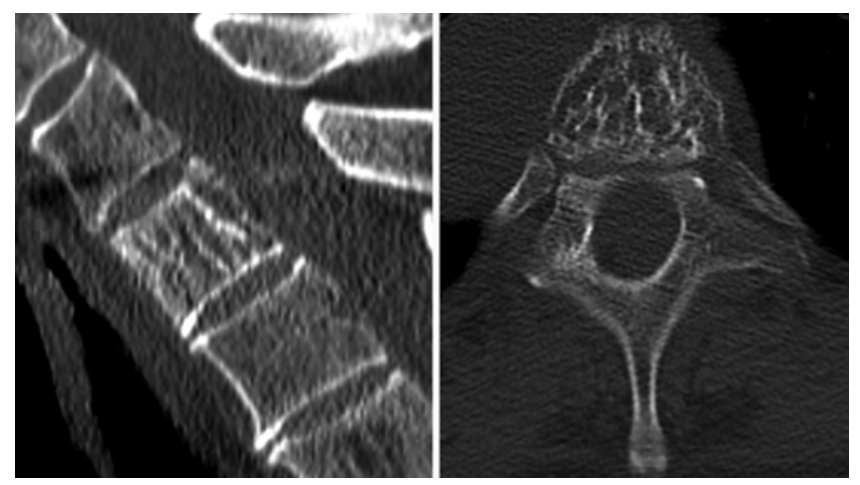

FIG. 1. Case 1. Computed tomography scans obtained prior to surgical decompression, showing the second thoracic vertebra, the vertical trabeculations on sagittal view (left), and the classic "polka dot" appearance of the enlarged vascular spaces of the patient's symptomatic vertebral hemangioma on axial view (right).

strategy for SVH, although this may be in part because of the limited evidence currently available. ${ }^{11}$ From our cohort, we noticed that partial improvement from SVH is achievable after SRS in the majority of patients. Our SRS candidates with SVH commonly underwent complex pain management before and after radiosurgery. Therefore, careful patient selection may be critical, and patients may require multiple treatment strategies for symptom control.

In monitoring the clinical response of patients, we see a positive response rate $(80 \%)$, with durable responses usually at 1 year. These rates and timelines are consistent with those in the existing radiotherapy literature..$^{14,19,23}$ Miszczyk and Tukiendorf reported complete pain relief in $64 \%$ of patients at 1.5 years compared with $36 \%$ at 1 month. ${ }^{18}$ This adds evidence to a hypothesis suggesting that SVH relief following SRS is more often a delayed outcome, unlike that seen in the prior SRS case report. ${ }^{18,25,27}$ Regarding who may benefit most from SRS, our 2 patients who underwent initial decompression experienced the best pain control, and patients receiving adjuvant SRS were likely to improve. More evidence, however, is necessary to validate these positive correlations, as the radiotherapy literature has not found prior surgery to be predictive of favorable outcomes. $^{4,14,25}$ In the meantime, the use of conformal radiation therapy offers a conservative means of treatment while avoiding the risk of watershed infarct in the midthoracic spine or retropulsion of a posterior lesion embolization or vertebroplasty. ${ }^{4,11}$

It is also very encouraging that an appreciable radiographic response can be achieved. Within our cohort, those who presented with reductions in lesion size were also noted to improve clinically. Favorable outcomes exhibited on follow-up images obtained after radiotherapy and SRS for the treatment of such vascular malformations have been correlated with clinical improvement, particularly in the intracranial lesion literature., ${ }^{914-16,21,23,25}$ However, the significance and likelihood of radiographic changes in the spine are still disputed. ${ }^{14,22}$ Predicting which lesions are most amenable to radiosurgical shrinkage should continue to be of ongoing interest for such vascular spinal lesions, as their extramedullary proximity to the spinal cord will be a major determinant of optimal SRS dosing when radiotherapy cannot achieve sufficient conformity. ${ }^{20}$
With regard to treatment planning for SVH, the SRS experience is still largely guided by the radiotherapy literature, and our series shows that current hypofractionated strategies remain consistently safe, without subsequent myelopathic injury or worsening of symptoms. ${ }^{27}$ Sohn et al. experienced success with administering $32 \mathrm{~Gy}$ in 4 fractions for a $2.4-\mathrm{cm}^{3}$ lesion. ${ }^{25}$ Our evidence demonstrates that SRS planning is feasible for much larger lesions and can achieve pain relief with fewer fractions. From the conventional fractionation literature, a higher dose, with a lower boundary of $35 \mathrm{~Gy}$, increased the likelihood of pain control. ${ }^{14,18,19}$ All patients from this series were safely administered an $\mathrm{nBED}$ greater than this recommended dose, adding continued evidence for this practice. For the 2 patients who exhibited regression in tumor size, both were treated with only 1 fraction, but with unrelated planning regimens, perhaps achieving a volumetric collapse given their large baseline sizes.

There exist significant limitations to our study. Namely, the small sample size precludes definitive conclusions regarding the efficacy of SRS. However, enough evidence exists to justify further investigation into larger controlled studies. Additional reporting of cases that build on our cohort will enable stratification of patients by their baseline symptom severities, comorbidities, and adjunct treatments to predict outcomes. The incorporation of postoperative and preoperative comprehensive pain assessments may also be valuable supplementary tools for trending the improvement of patients' complex symptoms following SRS.

\section{Conclusions}

Our clinical summaries suggest that some SVH patients present with complex comorbidities that potentially confound or reduce the benefit of any treatment response, radiosurgery or otherwise. It may be that a higher or complete response to primary SRS can be achieved in select patients who have clear, anatomically associated etiologies with susceptible tissue, thereby reducing multiple surgical procedures in nonemergent scenarios. Thus far, we have shown that SRS can be safely used as an adjunct in a multimodality approach to SVH and it can achieve appreciable success in large lesions.

\section{References}

1. Adler JR Jr, Murphy MJ, Chang SD, Hancock SL: Imageguided robotic radiosurgery. Neurosurgery 44:1299-1307, 1999

2. Ahn H, Jhaveri S, Yee A, Finkelstein J: Lumbar vertebral hemangioma causing cauda equina syndrome: a case report. Spine (Phila Pa 1976) 30:E662-E664, 2005

3. Aksu G, Korcum AF: Symptomatic vertebral hemangioma: results of radiotherapy. J Musculoskeletal Pain 16:318-325, 2009

4. Blecher R, Smorgick Y, Anekstein Y, Peer A, Mirovsky Y: Management of symptomatic vertebral hemangioma: followup of 6 patients. J Spinal Disord Tech 24:196-201, 2011

5. Bremnes RM, Hauge HN, Sagsveen R: Radiotherapy in the treatment of symptomatic vertebral hemangiomas: technical case report. Neurosurgery 39:1054-1058, 1996

6. Chang SD, Adler JR Jr: Current status and optimal use of radiosurgery. Oncology (Williston Park) 15:209-216, 219221,2001 
7. Chang SD, Main W, Martin DP, Gibbs IC, Heilbrun MP: An analysis of the accuracy of the CyberKnife: a robotic frameless stereotactic radiosurgical system. Neurosurgery 52:140-147, 2003

8. Chang UK, Rhee CH, Youn SM, Lee DH, Park SQ: Radiosurgery using the Cyberknife for benign spinal tumors: Korea Cancer Center Hospital experience. J Neurooncol 101:9199, 2011

9. Chou CW, Wu HM, Huang CI, Chung WY, Guo WY, Shih $\mathrm{YH}$, et al: Gamma Knife surgery for cavernous hemangiomas in the cavernous sinus. Neurosurgery 67:611-616, 2010

10. Faul CM, Flickinger JC: The use of radiation in the management of spinal metastases. J Neurooncol 23:149-161, 1995

11. Fox MW, Onofrio BM: The natural history and management of symptomatic and asymptomatic vertebral hemangiomas. J Neurosurg 78:36-45, 1993

12. Gerszten PC, Ozhasoglu C, Burton SA, Vogel WJ, Atkins BA, Kalnicki S, et al: CyberKnife frameless single-fraction stereotactic radiosurgery for benign tumors of the spine. Neurosurg Focus 14(5):e16, 2003

13. Grau SJ, Holtmannspoetter M, Seelos K, Tonn JC, Siefert A: Giant multilevel thoracic hemangioma with spinal cord compression in a patient with Klippel-Weber-Trenaunay syndrome: case report. Spine (Phila Pa 1976) 34:E498-E500, 2009

14. Heyd R, Seegenschmiedt MH, Rades D, Winkler C, Eich HT, Bruns F, et al: Radiotherapy for symptomatic vertebral hemangiomas: results of a multicenter study and literature review. Int J Radiat Oncol Biol Phys 77:217-225, 2010

15. Jones J, Jang S, Getch CC, Kepka AG, Marymont MH: Advances in the radiosurgical treatment of large inoperable arteriovenous malformations. Neurosurg Focus 23(6):E7, 2007

16. Kim MS, Pyo SY, Jeong YG, Lee SI, Jung YT, Sim JH: Gamma Knife surgery for intracranial cavernous hemangioma. J Neurosurg 102 (Suppl):102-106, 2005

17. Loblaw DA, Laperriere NJ: Emergency treatment of malignant extradural spinal cord compression: an evidence-based guideline. J Clin Oncol 16:1613-1624, 1998

18. Miszczyk L, Tukiendorf A: Radiotherapy of painful vertebral hemangiomas: the single center retrospective analysis of 137 cases. Int J Radiat Oncol Biol Phys 82:e173-e180, 2012

19. Rades D, Bajrovic A, Alberti W, Rudat V: Is there a doseeffect relationship for the treatment of symptomatic vertebral hemangioma? Int J Radiat Oncol Biol Phys 55:178-181, 2003

20. Rashad S, Endo T, Ogawa Y, Sato K, Endo H, Matsumoto Y, et al: Stereotactic radiosurgery as a feasible treatment for intramedullary spinal arteriovenous malformations: a singlecenter observation. Neurosurg Rev [epub ahead of print], 2016

21. Ryu SI, Chang SD, Kim DH, Murphy MJ, Le QT, Martin DP, et al: Image-guided hypo-fractionated stereotactic radiosurgery to spinal lesions. Neurosurgery 49:838-846, 2001

22. Sakata K, Hareyama M, Oouchi A, Sido M, Nagakura H, Tamakawa M, et al: Radiotherapy of vertebral hemangiomas. Acta Oncol 36:719-724, 1997

23. Schild SE, Buskirk SJ, Frick LM, Cupps RE: Radiotherapy for large symptomatic hemangiomas. Int J Radiat Oncol Biol Phys 21:729-735, 1991

24. Serrano M, Iglesias A, San Millan J: Long-term response to radiotherapy of vertebral hemangioma resulting in paraplegia. Acta Oncol 35:498-499, 1996

25. Sohn MJ, Lee DJ, Jeon SR, Khang SK: Spinal radiosurgical treatment for thoracic epidural cavernous hemangioma presenting as radiculomyelopathy: technical case report. Neurosurgery 64:E1202-E1203, 2009

26. Templin CR, Stambough JB, Stambough JL: Acute spinal cord compression caused by vertebral hemangioma. Spine J 4:595-600, 2004

27. Yang ZY, Zhang LJ, Chen ZX, Hu HY: Hemangioma of the vertebral column. A report on twenty-three patients with special reference to functional recovery after radiation therapy. Acta Radiol Oncol 24:129-132, 1985

\section{Disclosures}

The authors report no conflict of interest concerning the materials or methods used in this study or the findings specified in this paper.

\section{Author Contributions}

Conception and design: all authors. Acquisition of data: Zhang, Chang. Analysis and interpretation of data: Zhang, Chen. Drafting the article: Zhang. Critically revising the article: Veeravagu, Zhang, Chen. Reviewed submitted version of manuscript: Zhang, Chen, Chang. Statistical analysis: Zhang. Administrative/techni$\mathrm{cal} / \mathrm{material}$ support: Zhang, Chang. Study supervision: Veeravagu, Chen, Chang.

\section{Correspondence}

Anand Veeravagu, Department of Neurosurgery, Stanford University, 300 Pasteur Dr., Edwards Bldg. R-292, Stanford, CA 943055327. email: anand.veeravagu@stanford.edu. 